Rev. Int. Contam. Ambie. 33 (3) 495-503, 2017

DOI: 10.20937/RICA.2017.33.03.12

\title{
FITORREMEDIACIÓN DE UN SUELO CONTAMINADO CON PETRÓLEO EXTRA PESADO CON Megathyrsus maximus
}

\author{
Ismael HERNÁNDEZ VALENCIA ${ }^{1 *}$, Gabriela NAVAS ${ }^{2}$ y Carmen INFANTE $^{3}$
}

${ }^{1}$ Centro de Ecología Aplicada, Instituto de Zoología y Ecología Tropical, Universidad Central de Venezuela. Apartado Postal 47058, Caracas 1041-A, Venezuela

${ }^{2}$ Escuela de Biología, Facultad de Ciencias, Universidad Central de Venezuela. Apartado Postal 47114, Caracas 1041-A, Venezuela

${ }^{3}$ Postgrado de Geoquímica, Facultad de Ciencias, Universidad Central de Venezuela. Apartado Postal 3895, Caracas 1041-A, Venezuela

*Autor para correspondencia: ismael.hernandez@ciens.ucv.ve

(Recibido abril 2016; aceptado noviembre 2016)

Palabras clave: contaminación, biorremediación, pastos, actividad microbiana, trópico

\section{RESUMEN}

La contaminación del suelo por petróleo es un problema ambiental y la fitorremediación ha probado ser una técnica promisoria para recuperar estos suelos. No obstante, la selección de especies para ser usadas en la fitorremediación es clave para obtener resultados satisfactorios. En el presente trabajo se estudió la capacidad del pasto Megathyrsus maximus para fitorremediar un suelo contaminado con petróleo extra pesado, a través de los cambios durante 120 días en el contenido de hidrocarburos totales de petróleo y de indicadores de la actividad microbiológica en el suelo (actividad de la enzima deshidrogenasa, biomasa del carbono microbiano y respiración basal), los cuales fueron evaluados en dos tratamientos, uno en suelos contaminados en donde se trasplantó Megathyrsus maximus y otro en suelos contaminado sin la planta. Las diferencias entre tratamientos fueron pequeñas pero significativas. Los resultados mostraron que en 120 días el contenido de hidrocarburos se redujo en un $17.1 \%$, en el tratamiento con M. maximus y en $9.8 \%$ en el tratamiento sin plantas. Las fracciones de los hidrocarburos que disminuyeron fueron las de aromáticos y saturados para el tratamiento con M. maximus y la de aromáticos para el tratamiento sin plantas. El tratamiento con pastos mostró cambios en el tiempo para todos los indicadores bioquímicos y microbiológicos evaluados, mientras que el tratamiento sin pastos sólo para la actividad de la enzima deshidrogenasa y el coeficiente metabólico. La actividad microbiana fue siempre mayor o similar en el tratamiento con plantas, probablemente por la presencia de una rizósfera que favorece dicha actividad.

Key words: pollution, bioremediation, pastures, microbial activity, tropic

\begin{abstract}
Soil contamination by petroleum is an environmental problem and phytoremediation has proved to be a promising method for treating such soils. However, selection of species for use in phytoremediation is a key factor for its successful application. The aim of
\end{abstract}


this study was to investigate the phytoremediation potential of the grass Megathyrsus maximus in heavy crude-oil contaminated soils. Temporal changes on total hydrocarbons and soil microbiological activity (enzyme activity of dehydrogenase, microbial biomass carbon and basal respiration) were evaluated in two treatments of oil contaminated soil, planted and unplanted with M maximus, over a 120-day period. Differences between both treatments were small but significant. Hydrocarbon concentration was reduced $17.1 \%$ in planted soils and $9.8 \%$ in unplanted treatments. Both saturated and aromatic hydrocarbon fractions were reduced in planted treatments but aromatics was the only fraction reduced in unplanted treatment. Biochemical and microbiological indicators varied temporally in the planted treatment but the unplanted treatment only showed differences for dehydrogenase enzyme activity and the metabolic coefficient. Microbial activity was always higher or similar in the planted treatment compared to the unplanted one, probably as a result of the rhizosphere favoring this activity.

\section{INTRODUCCIÓN}

La contaminación de los suelos por derrames o disposición inadecuada de petróleo es un problema ambiental mundial, particularmente la contaminación producida por el petróleo pesado y extra pesado, que aunque son menos tóxicos que los petróleos livianos y medianos, su remediación es más difícil y costosa (Infante y Morales 2012). Adicionalmente, aún cuando la toxicidad de los petróleos pesados y extra pesados suele ser menor, existe un efecto físico sobre las propiedades del suelo, como es la disminución de la capacidad de campo e incremento de la repelencia al agua, entre otras propiedades, que merman su fertilidad (Adams et al. 2008). Cabe destacar que el bitumen y los hidrocarburos pesados constituyen las mayores reservas de petróleo del mundo (Head et al. 2014), hecho que justifica la investigación sobre la rehabilitación de suelos contaminados por este tipo de hidrocarburos.

Dentro de las técnicas para la descontaminación de suelos, la fitorremediación resulta atractiva por su bajo costo, por mejorar las condiciones del suelo, por ser estéticamente agradable y ambientalmente amigable (Pilon 2005, Yavari et al. 2015). La fitorremediación se fundamenta en el uso de plantas y su microbiota asociada para remover, retener o reducir los contaminantes presentes en el ambiente y puede operar mediante diversos mecanismos que involucran distintas partes de las plantas (Chávez-Maldonado et al. 2010, Khan et al. 2013). Entre los mecanismos asociados a la fitorremediación se pueden mencionar a la fitoextracción, la fitoestabilización, la fitovolatilización y la rizodegradación, siendo esta última reconocida como la principal responsable de la disminución de hidrocarburos de petróleo en el suelo (Yavari et al. 2015). La rizodegradación se basa en el fomento de la actividad microbiana asociada a la rizósfera para degradar los compuestos carbonados que se encuentran en ella. Cabe destacar que los exudados de las raíces contienen aminoácidos, ácidos orgánicos, carbohidratos, factores de crecimiento y proteínas solubles que incrementan la actividad microbiana en la rizósfera (Miya y Firestone 2001).

La contribución de las plantas y los microorganismos en la fitorremediación de suelos contaminados con petróleo, puede ser evaluada a través de indicadores de la actividad microbiológica del suelo, como son la respiración basal, la actividad de la enzima deshidrogenasa y la biomasa del carbono microbiano, ya que estos indicadores están relacionados con el aprovechamiento, por parte de los microorganismos, de los compuestos de carbono en el suelo bajo condiciones aeróbicas (Mager y Hernández-Valencia 2013). Se parte de la premisa que la adición de hidrocarburos al suelo incrementará la actividad microbiana al contar los microorganismos con una fuente de carbono y energía en el petróleo. Adicionalmente, la actividad microbiana, así como la degradación de hidrocarburos, será aún mayor en los suelos con plantas por la presencia de una rizósfera que estimula esta actividad.

Con base en lo expuesto, en este estudio se evaluaron los cambios producidos en la actividad microbiana durante la fitorremediación con el pasto Megathyrsus máximus de un suelo contaminado con petróleo extra pesado de $9^{\circ}$ de acuerdo con la clasificación del American Petroleum Institutue (API). Esta especie fue seleccionada ya que está adaptada a ambientes tropicales, presenta rápido crecimiento, sus requerimientos agronómicos son ampliamente conocidos, sus semillas se consiguen fácilmente en los comercios agrícolas y es muy frecuente en hábitats perturbados. Por otra parte, su potencial para fitorremediar suelos contaminados con petróleo liviano ha sido probado (Mager y Hernández-Valencia 2013). 


\section{MATERIALES Y MÉTODOS}

\section{Diseño experimental y preparación de muestras}

El suelo usado en los ensayos fue obtenido del horizonte A de un haplustox típico, franco arenoso, fuertemente ácido y pobre en nutrimentos (Hernández-Valencia y Mager 2003), proveniente de la localidad de El Sombrero, estado Guárico, Venezuela. Por su parte, el petróleo utilizado presentó una gravedad específica de $9^{\circ} \mathrm{API}$, por lo cual se le clasifica como extra pesado. Como planta con potencial fitorremediador se usó al pasto Megathyrsus maximus (Jacq.) B. K. Simon y S. W. L. Jacobs (guinea, gamelote), una gramínea perenne, de rápido crecimiento, introducida de África, pero de amplia distribución en Venezuela. Se utilizaron plantas con alturas entre 25-30 cm, obtenidas a partir de la germinación de semillas certificadas en suelos no contaminados.

Se establecieron dos tratamientos en condiciones de invernadero: a) suelo contaminado con $50.0 \mathrm{mg} / \mathrm{g}$ de petróleo extra pesado y con el pasto (CP), b) suelo contaminado con $50.0 \mathrm{mg} / \mathrm{g}$ de petróleo extra pesado sin el pasto (SP). El suelo contaminado fue una mezcla preparada con $85 \%$ de suelo, $5 \%$ de petróleo extra pesado y $10 \%$ de cáscara de arroz $(\mathrm{m} / \mathrm{m})$ como agente estructurante, con mayor capacidad de retención de agua, y de mejorar la actividad microbiana (Infante et al. 2012). Una cantidad de 2.5 $\mathrm{kg}$ de la mezcla de suelo, petróleo y cáscara de arroz fue colocada en envases cilíndricos de policloruro de vinilo (PVC) de $30 \mathrm{~cm}$ de alto y $10 \mathrm{~cm}$ de diámetro. En el tratamiento con el pasto se sembró un individuo de $M$. maximus por envase. Las condiciones microclimáticas promedio del invernadero durante el ensayo fueron $23.2{ }^{\circ} \mathrm{C}$ de temperatura, $450 \mu \mathrm{E} / \mathrm{s}$ de radiación y $85 \%$ de humedad relativa. Para cada tratamiento se establecieron cinco réplicas y la duración del experimento fue de 120 días.

Todos los tratamientos fueron fertilizados con $\mathrm{N}: \mathrm{P}: K:(15: 15: 15)$ y sulfato de amonio, en una relación C:N:P de 100:2:0.2, similar a la utilizada por Hutchinson et al. (2001). El fertilizante se incorporó un $50 \%$ (1.772 g de N:P:K y $4.264 \mathrm{~g}$ de sulfato de amonio por envase) al inicio del ensayo, y el restante $50 \%$ fue repartido equitativamente a los 30 y 60 días siguientes. Los envases fueron regados cada $5 \mathrm{~d}$ con agua del servicio doméstico, manteniendo el suelo a $50 \%$ de su capacidad de campo. La capacidad de campo fue estimada por triplicado en una masa de suelo previamente contaminada con el petróleo extra pesado a la concentración de $50 \mathrm{mg} / \mathrm{g}$, al cual se agregó agua hasta su saturación. Luego el suelo fue cubierto con un manto plástico para reducir las pérdidas de agua por evaporación y posteriormente se dejó drenar el excedente de agua durante $72 \mathrm{~h}$. El agua retenida o capacidad de campo se estimó como la diferencia de masa entre el suelo húmedo luego de $72 \mathrm{~h}$ y el suelo seco, después de ser secado por $48 \mathrm{~h}$ a $105^{\circ} \mathrm{C}$ en una estufa (Anderson e Ingram 1992).

\section{Determinación de hidrocarburos del petróleo y sus fracciones}

Se colectaron muestras de los suelos de ambos tratamientos a los $0,15,30,60$ y $120 \mathrm{~d}$ de iniciado el ensayo para realizar determinaciones del contenido de hidrocarburos por el método EPA 3540C (USEPA 1996), empleando como agente extractante diclorometano. Adicionalmente, a los extractos de hidrocarburos obtenidos al inicio y al final del ensayo de cada tratamiento se les hizo un fraccionamiento para determinar las variaciones en el contenido de saturados, aromáticos, resinas y asfaltenos (SARA), de acuerdo con el método 5520 (APHA 1998), procedimiento que se basa en la separación de las diferentes fracciones del petróleo a través de técnicas de precipitación y adsorción, según la solubilidad y polaridad de dichas fracciones.

\section{Determinación de indicadores de la actividad microbiológica en el suelo}

Para las muestras de suelos obtenidas en cada muestreo se determinó la actividad de la enzima deshidrogenasa (ADH), el carbono de la biomasa microbiana (Cmic) y la respiración basal (RB). La actividad de la enzima deshidrogenasa se determinó según el método de Casida et al. (1964), el cual se basa en la reducción del cloruro de trifenil tetrazolio (TTC) por la acción de dicha enzima a trifenil formazan (TPF), lo que origina un precipitado de color rojo soluble en metanol. Se emplearon $1.5 \mathrm{~g}$ suelo en peso fresco que se colocaron en tubos de ensayo con $0.25 \mathrm{~mL}$ de TTC al $3 \%, 0.05 \mathrm{~g}$ de $\mathrm{CaCO}_{3}$ y $2.5 \mathrm{~mL}$ de agua destilada. Las muestras se incubaron por $24 \mathrm{~h}$ a $37^{\circ} \mathrm{C}$ y posteriormente se añadieron $2.5 \mathrm{~mL}$ de $\mathrm{CH}_{3} \mathrm{OH}$. Finalmente la muestra se filtró y se midió la absorbancia a $485 \mathrm{~nm}$. Los patrones fueron preparados a partir de una solución estándar de TPF de $1 \mathrm{mg} / \mathrm{L}$.

Para la determinación del Cmic se empleó el método de fumigación-extracción según Vance et al. (1987) que estima este indicador como el contenido de carbono soluble en una solución de $\mathrm{K}_{2} \mathrm{SO}_{4} 0.5 \mathrm{M}$. Para ello se utilizaron muestras de $5 \mathrm{~g}$ de suelo a $50 \%$ de su capacidad de campo, por sextuplicado para cada serie de tiempo. Tres réplicas se fumigaron con cloroformo libre de etanol en una cámara de vacío 
por $24 \mathrm{~h}$ con el fin de provocar la muerte de las células microbianas, mientras que las tres réplicas restantes no fueron fumigadas. El carbono de las muestras fumigadas y no fumigadas se extrajo con $40 \mathrm{~mL}$ de $\mathrm{K}_{2} \mathrm{SO}_{4} 0.5 \mathrm{M}$ agitando durante $1 \mathrm{~h}$. Posteriormente se tomaron $8 \mathrm{~mL}$ del extracto y se añadieron $15 \mathrm{~mL}$ de una mezcla ácida ( $2 / 3$ partes de $\mathrm{H}_{2} \mathrm{SO}_{4}$ y $1 / 3$ de $\mathrm{H}_{3} \mathrm{PO}_{4}$ ), 2 mL de $\mathrm{K}_{2} \mathrm{Cr}_{2} \mathrm{O}_{7} 66.7 \mathrm{mM}$ y $70 \mathrm{mg}$ de $\mathrm{HgO}$. La mezcla se colocó a digestión durante 30 min a $150^{\circ} \mathrm{C}$ y luego se valoró con sulfato amónico ferroso $33 \mathrm{mM}$ empleando como indicador 1.10 fenantrolinaferrosa $25 \mathrm{mM}$. La cantidad de carbono de la biomasa microbiana se determinó mediante la fórmula:

$\mathrm{Cmic}=(\mathrm{C}$ orgánico fumigado -

$\mathrm{C}$ orgánico no fumigado $/ \mathrm{K}_{\mathrm{ec}}$

Donde $\mathrm{K}_{\mathrm{ec}}=0.38$, es una constante de recuperación calculada a partir de la correlación de los resultados obtenidos en doce suelos diferentes en donde se utilizó este método (Vance et al. 1987).

La respiración basal o $\mathrm{CO}_{2}$ liberado por los microorganismos fue estimado por el método de Alef y Nannipieri (1995). Para cada tiempo se utilizaron $25 \mathrm{~g}$ de suelo al $50 \%$ de capacidad de campo y se colocaron en envases de plástico con $50 \mathrm{~mL}$ de capacidad. En envases similares se colocaron $10 \mathrm{~mL}$ de $\mathrm{NaOH} 0.5$ $\mathrm{M}$ como solución que absorbe el $\mathrm{CO}_{2}$ emitido por los microorganismos. Ambos envases se colocaron dentro de un recipiente de vidrio herméticamente cerrado y fueron incubados en la oscuridad a $23{ }^{\circ} \mathrm{C}$ durante $48 \mathrm{~h}$. Transcurrido el tiempo de incubación se añadió a cada envase $1 \mathrm{~mL}$ de $\mathrm{BaCl}_{2} 0.5 \mathrm{M}$ para precipitar como carbonato al $\mathrm{CO}_{2}$ fijado y el $\mathrm{NaOH}$ restante se valoró con $\mathrm{HCl} 0.5 \mathrm{M}$, utilizando como indicador fenolftaleina al $1 \%$, disuelto en $\mathrm{CH}_{3} \mathrm{COH}$. Con los datos de respiración basal y Cmic se calculó el coeficiente metabólico $\left(\mathrm{qCO}_{2}=\mathrm{RB} / \mathrm{Cmic}\right)$, que es una medida de la eficiencia con la cual los microorganismos utilizan el carbono disponible para la biosíntesis (Marín et al. 2005).

Los resultados de los indicadores antes mencionados fueron expresados con base en el peso seco, luego de secar triplicados de las muestras de suelo en una estufa a $105^{\circ} \mathrm{C}$ por $48 \mathrm{~h}$ y pesadas en una balanza de $0.01 \mathrm{~g}$ de precisión.

\section{Análisis estadísticos}

Las medias de los tratamientos de los diferentes parámetros evaluados fueron comparadas a través de un análisis de varianza de dos vías y la prueba a posteriori de la mínima diferencia significativa (LSD, por sus siglas en inglés) de Fisher con un nivel de significancia del 0.05 . También se realizaron pruebas de correlación lineal de las variaciones en el tiempo entre el contenido de hidrocarburos, respiración basal, Cmic, coeficiente metabólico y actividad de la enzima deshidrogenasa. Para todas las pruebas estadísticas se usó el programa Statistica 8.0 (Statsoft 2007).

\section{RESULTADOS Y DISCUSIÓN}

\section{Contenido total y fraccionamiento de los hidro- carburos en el suelo}

La figura 1 muestra los cambios en el contenido de hidrocarburos en el tiempo. Se debe mencionar que este método permite determinar tanto los hidrocarburos de tipo mineral, como vegetal y animal. Sin embargo, en vista de que análisis previos mostraron que el contenido de aceites y grasas en suelos no contaminados fue inferior a $5 \mathrm{mg} / \mathrm{g}$, se desestimó la contribución de la fracción vegetal y animal, por lo que en consecuencia nos referiremos a los aceites y grasas extraídos en este estudio como a hidrocarburos del petróleo.

Destaca que la recuperación del petróleo del suelo no fue total; es decir, de la mezcla originalmente preparada de $50.0 \mathrm{mg} / \mathrm{g}$ de petróleo extra pesado, se obtuvo una concentración inicial de hidrocarburos del $37.5 \mathrm{mg} / \mathrm{g}$, lo que implica una recuperación del $75 \%$ aproximadamente. Este porcentaje de recuperación es similar a otros reportes en la literatura (Infante et al. 2012, Mager y Hernández Valencia 2013), y se asocia con que el extractante no logra la desadsorción completa del petróleo por las interacciones con los componentes de mayor polaridad con la matriz del

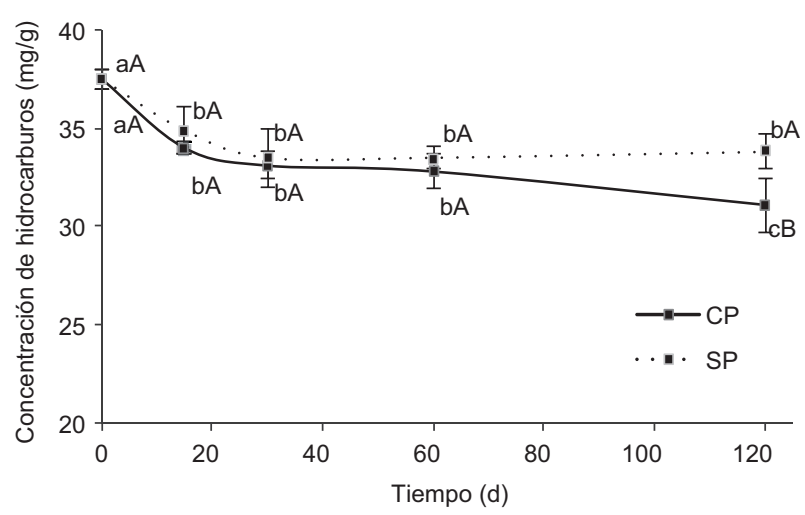

Fig. 1. Variación en el tiempo del contenido de hidrocarburos de petróleo. Letras minúsculas iguales indican que no hay diferencias para un mismo tratamiento en diferentes tiempos. Letras mayúsculas iguales indican que no hay diferencias entre tratamientos para un mismo tiempo. Las barras corresponden a la desviación estándar $(n=5)$ 
suelo y por la volatilización hidrocarburos de bajo peso molecular (Infante et al. 2012, Córdova et al. 2013). Con base en ello, se usó para los cálculos de disminución de la concentración de hidrocarburos en el tiempo, un valor inicial de $37.5 \mathrm{mg} / \mathrm{g}$ y no de $50.0 \mathrm{mg} / \mathrm{g}$

Durante los 120 días del ensayo, el valor inicial de $37.5 \mathrm{mg} / \mathrm{g}$ de hidrocarburos disminuyó a $33.8 \mathrm{mg} / \mathrm{g}$ en SP y $31.1 \mathrm{mg} / \mathrm{g}$ en CP, siendo estadísticamente diferentes entre sí sólo a los 120 d (Fig.1). La mayor disminución del contenido de hidrocarburos en los primeros $15 \mathrm{~d}$ para ambos tratamientos se relaciona con la rápida degradación de los componentes más lábiles, como son los saturados y los aromáticos, las fracciones más biodegradables, pero también más toxicas respecto a las resinas y asfaltenos (Ehrmann et al. 2009, Infante y Morales 2012). Posteriormente, a partir de los $15 \mathrm{~d}$ y hasta el término del ensayo, el tratamiento SP no evidenció una reducción significativa en el contenido de hidrocarburos.

La dinámica en el tiempo del contenido de hidrocarburos en el suelo para ambos tratamientos se ajustó a una curva exponencial negativa del tipo:

$\mathrm{Pt}=\mathrm{Po} \mathrm{e}^{-\mathrm{kt}}$

en donde Po y Pt es la masa inicial y remanente de hidrocarburos respectivamente, $t$ es el tiempo transcurrido y k la constante de pérdida (Mager y Hernández Valencia 2013). La ecuación anterior puede ser expresada también como:

$\ln (\mathrm{Pt} / \mathrm{Po})=-\mathrm{kt}$

Cuando los datos se ajustan al modelo, describen una recta cuya pendiente $(\mathrm{k})$ es la constante de pérdida de los hidrocarburos. Una ventaja del ajuste de los datos a este modelo, es que permite calcular también el tiempo de vida media $(0.693 / \mathrm{k})$, así como la tasa de recambio o el tiempo requerido para que se degrade el $99 \%(4.605 / \mathrm{k})$ del contenido original de hidrocarburos (Mager y Hernández Valencia 2013).

Sólo los datos de la variación en el tiempo en el contenido de hidrocarburos en $\mathrm{CP}$ se ajustaron al modelo exponencial negativo $(\mathrm{r}=-0.88, \mathrm{p}=0.05$, $\mathrm{n}=5$ ). La constante de degradación ( $\mathrm{k}$ ) fue de -0.46 , lo que permite estimar que bajo las condiciones de este tratamiento, la vida media y la tasa de recambio del petróleo utilizado son de 1.5 y 10.7 años respectivamente. La baja tasa de biodegradación de este tipo de petróleo se confirma cuando se contrasta con los resultados obtenidos por Mager y HernándezValencia (2013), quienes encontraron para la misma especie y suelos contaminados a $30.0 \mathrm{mg} / \mathrm{g}$ con un petróleo más liviano, de $27.2^{\circ} \mathrm{API}$, que el tiempo de vida media y la tasa de recambio fueron de 0.5 y 3.1 años respectivamente. Evidentemente, las diferencias de estos resultados con los de nuestra investigación podrían justificarse por el hecho de que usamos un petróleo extra pesado, con mayor contenido de compuestos resistentes a la biodegradación, como resinas y asfaltenos, además en una concentración inicial en el suelo más alta, y en consecuencia un potencial más tóxico.

Respecto al uso de $M$. maximus en la recuperación de suelos contaminados con petróleo extra pesado, pese a que se encontraron diferencias significativas en los tratamientos con pastos a los $120 \mathrm{~d}$, estas no fueron muy grandes respecto al tratamiento sin plantas, hecho que indica que en este caso el uso de fitorremediación no es tan eficiente, y en consecuencia pudieran proveer mejores resultados otras técnicas biológicas como la biorremediación con técnicas agrícolas (landfarming, en inglés) y el compostaje (Infante et al. 2010a) e incluso en otras basadas en procesos físicos o químicos (incineración, lavado, uso de surfactantes) (Yavari et al. 2015). Sin embargo, el uso de uso de M. maximus puede ser ventajoso si se implementa luego de usar estas técnicas, ya que el establecimiento de plantas con fines de fitorremediación mejora la calidad del suelo al aportar materia orgánica y fomentar la actividad biológica, además de remover compuestos contaminantes remanentes, proteger al suelo contra la erosión y procurar desde el punto de vista estético un entorno más agradable. Estos aspectos requieren mayores estudios en el futuro.

Dentro de las fracciones que componen los hidrocarburos extra pesados, las fracciones de saturados y aromáticos para $\mathrm{CP}$ y sólo la de aromáticos para SP disminuyeron a los $120 \mathrm{~d}$ (Fig. 2). Las resinas y los asfaltenos no mostraron cambios significativos, lo cual se asocia a su reconocida baja biodegradabilidad (Moubasher et al. 2015). Se debe destacar que los aromáticos forman parte de las fracciones más tóxicas del petróleo (Płaza et al. 2005). Por lo que su disminución es beneficiosa para el suelo y el ambiente en general. Dentro de la gama de compuestos aromáticos se encuentran los denominados hidrocarburos aromáticos policíclicos, siendo algunos de ellos cancerígenos (Rengarajan et al. 2015).

\section{Respiración basal, actividad de la enzima deshi- drogenasa y carbono de la biomasa microbiana}

A $\operatorname{los} 0,15,30,60$ y $120 \mathrm{~d}$ no se registraron diferencias significativas en la respiración basal entre los tratamientos con y sin plantas (Fig. 3) y ello se 


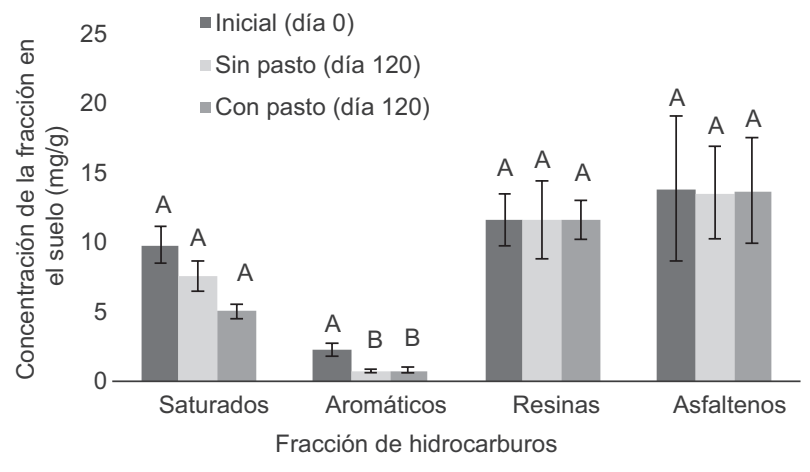

Fig. 2. Concentraciones iniciales y finales de las fracciones de aromáticos, saturados, resinas y asfaltenos en suelos contaminados con petróleo extra pesado. Letras minúsculas iguales indican que no hay diferencias para una fracción en los diferentes tiempos. Las barras corresponden a la desviación estándar $(\mathrm{n}=5)$

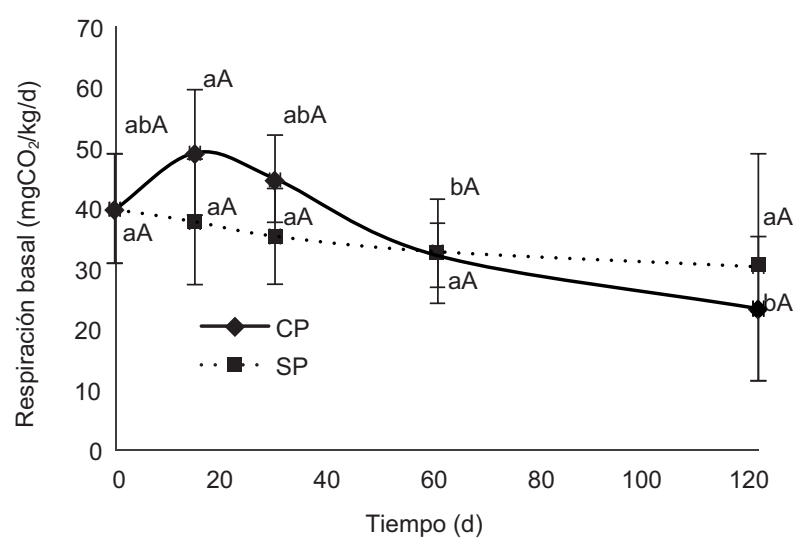

Fig. 3. Variaciones de la respiración basal. Letras minúsculas iguales indican que no hay diferencias para un mismo tratamiento en diferentes tiempos. Letras mayúsculas iguales indican que no hay diferencias entre tratamientos para un mismo tiempo. Las barras corresponden a la desviación estándar $(\mathrm{n}=5)$

asocia a la alta variabilidad de los datos. Resultados similares fueron obtenidos por Besalatpour et al. (2010), donde la presencia de Carthamus tinctorius, en suelos contaminados con $43.0 \mathrm{mg} / \mathrm{g}$ de petróleo no incrementó la producción de $\mathrm{CO}_{2}$, aunque sí lo hizo en presencia de Agropyron smithii, Festuca arundinacea y Helianthus annus.

En CP la respiración basal fue mayor a los $15 \mathrm{~d}$ respecto a los $120 \mathrm{~d}$. Lo que sugiere que la actividad de los microorganismos se ve inicialmente favorecida por la presencia de raíces, sus exudados y el carbono proveniente de los hidrocarburos. Posteriormente tiende a disminuir en la medida que lo hacen los hidrocarburos, especialmente aquellas fracciones biodegradables, hecho ya reportado por otros autores en diferentes suelos y tipos de hidrocarburos sin plantas (Infante et al. 2010b, Riveroll-Larios et al. 2015). En el caso de SP no se encontraron cambios significativos en la respiración basal a los $0,15,30$, 60 y $120 \mathrm{~d}$.

El contenido de carbono de la biomasa microbiana no mostró diferencias entre tratamientos los primeros 30 d. Posteriormente, en CP acusó un incremento a los 60 y $120 \mathrm{~d}$, pero sólo a los $60 \mathrm{~d}$, el carbono de la biomasa microbiana fue superior al registro de SP. Por otra parte, SP se mantuvo sin cambios significativos durante el desarrollo de los experimentos (Fig. 4).

Los resultados del coeficiente metabólico $\left(\mathrm{qCO}_{2}\right)$ indican que al inicio los microorganismos presentaron la mayor tasa de emisión de $\mathrm{CO}_{2}$ por masa de $\mathrm{C}$ microbiano y posteriormente esta disminuye en el tiempo (Fig. 5). En los primeros 30 $\mathrm{d}$ no se registraron diferencias en el $\mathrm{qCO}_{2}$ de ambos tratamientos. Entre los días 60 y 120 el $\mathrm{qCO}_{2}$ fue menor en el tratamiento con plantas, lo que pudiera indicar una condición ambiental menos estresante, ligada a la disminución del contenido de hidrocarburos y de los compuestos más tóxicos como los aromáticos y los saturados (Figs. 1 y 2). Hecho que reduce los requerimientos energéticos y de carbono para la sobrevivencia y crecimiento de la biomasa microbiana (Caravaca y Roldán 2003).

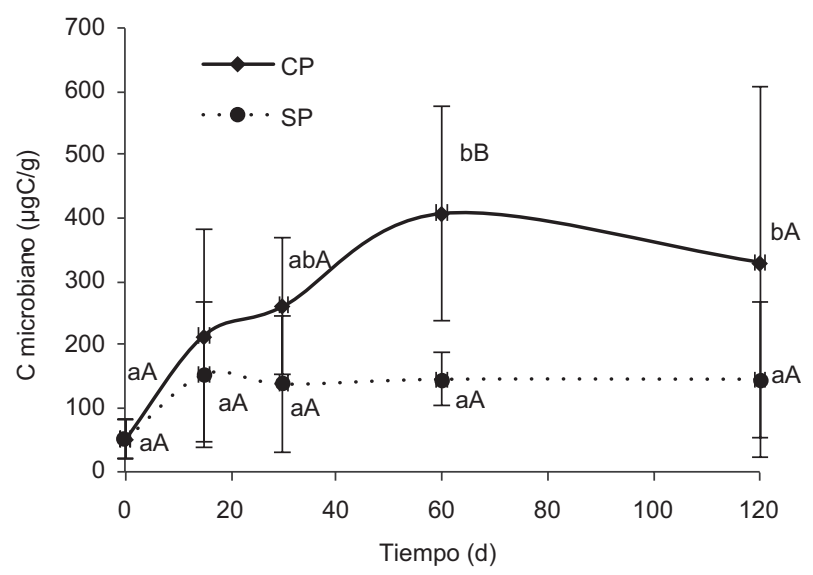

Fig. 4. Variaciones del carbono $(\mathrm{C})$ microbiano. Letras minúsculas iguales indican que no hay diferencias para un mismo tratamiento en diferentes tiempos. Letras mayúsculas iguales indican que no hay diferencias entre tratamientos para un mismo tiempo. Las barras corresponden a la desviación estándar $(\mathrm{n}=5)$

La actividad de la enzima deshidrogenasa (ADH) mostró un pico para CP y SP a los 30 d (Fig. 6). Sin embargo sólo se encontraron diferencias significativas entre tratamientos a los 15 y $60 \mathrm{~d}$, siendo mayor la ADH en CP. El incremento de la ADH los 


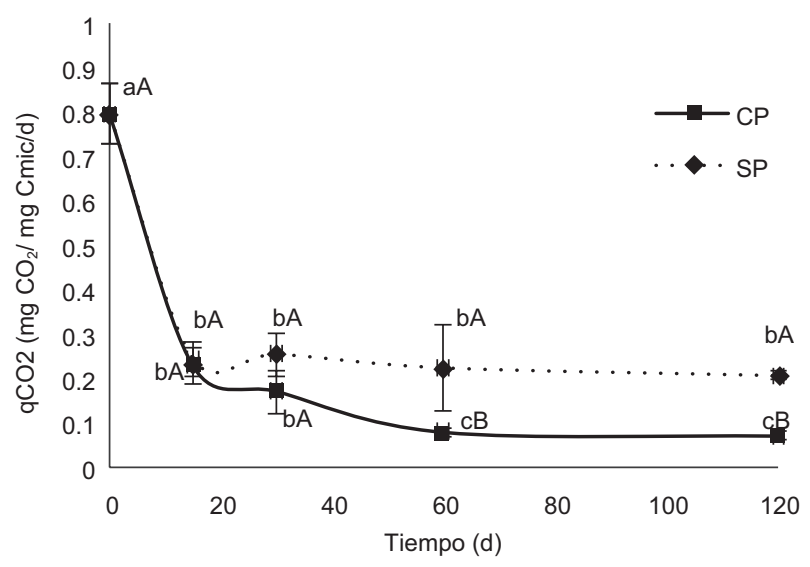

Fig. 5. Variaciones del coeficiente metabólico $\left(\mathrm{qCO}_{2}\right)$. Letras minúsculas iguales indican que no hay diferencias para un mismo tratamiento en diferentes tiempos. Letras mayúsculas iguales indican que no hay diferencias entre tratamientos para un mismo tiempo. Las barras corresponden a la desviación estándar $(\mathrm{n}=5)$

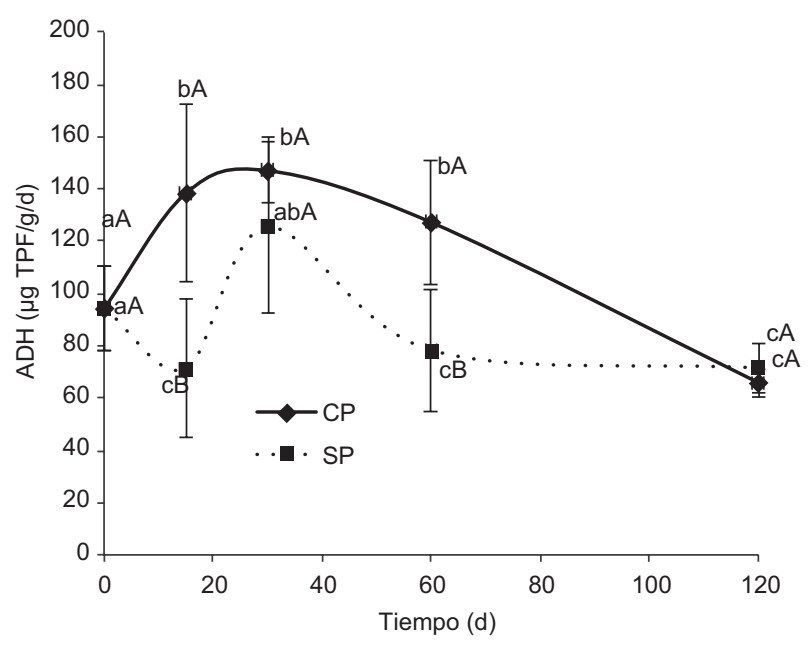

Fig. 6. Variaciones en la actividad de la enzima deshidrogenasa. Letras minúsculas iguales indican que no hay diferencias para un mismo tratamiento en diferentes tiempos. Letras mayúsculas iguales indican que no hay diferencias entre tratamientos para un mismo tiempo. Las barras corresponden a la desviación estándar $(\mathrm{n}=5) . \mathrm{TPF}=$ Trifenil formazán

primeros $30 \mathrm{~d}$ se asocia al aprovechamiento del carbono del petróleo, especialmente aquellas fracciones más biodegradables como los aromáticos y los saturados. Posteriormente, a partir del día 60 , con la reducción de las fracciones más biodegradables y la persistencia de las formas más recalcitrantes de los hidrocarburos, disminuyó la actividad de la enzima deshidrogenasa. Mager y Hernández Valencia (2013), señalan un comportamiento similar para la misma especie, pero en suelos contaminados con petróleo liviano.
Las correlaciones lineales entre los diferentes parámetros evaluados sólo fueron significativas y de manera inversa entre el contenido de hidrocarburos y el Cmic $\left(r^{2}=-0.66\right)$ y de manera directa entre el contenido de hidrocarburos y el $\mathrm{qCO}_{2}\left(\mathrm{r}^{2}=0.97\right)$. Ello sugiere que: a) la biomasa microbiana incrementa a expensas de la degradación del petróleo y eventualmente de la disminución de las fracciones más tóxicas como los saturados y los aromáticos (Fig. 2) y b) con la reducción del contenido de hidrocarburos y de los compuestos más tóxicos, el ambiente del suelo es menos estresante para los microorganismos, y con ello los requerimientos de mantenimiento de la biomasa microbiana, lo que se traduce en una menor respiración por biomasa de microorganismos (Marín et al 2005).

Los resultados obtenidos muestran que M. maximus favorece la reducción del contenido de hidrocarburos en el suelo en comparación con el tratamiento sin plantas. No obstante, las diferencias son visibles a partir de los 120 d. En lapsos de tiempo menores, la planta no ejerce cambios significativos. La tasa de degradación de los hidrocarburos se puede considerar baja, tal como se esperaría para un petróleo extra pesado. Si se toma en cuenta que las fracciones de aromáticos y saturados son las más biodegradables y que el contenido inicial de estas fracciones de acuerdo con los resultados del fraccionamiento de SARA es de $32 \%$ (Fig. 2), se podría disminuir aún más la concentración de hidrocarburos en un ensayo de mayor duración. En este estudio de 120 d la reducción del contenido de hidrocarburos y su desviación standard fue de $9.8 \% \pm 3.9 \%$ para SP y $17.1 \% \pm 3.2 \%$ para CP.

En cuanto al comportamiento de los parámetros microbiológicos y bioquímicos seleccionados como indicadores de la actividad microbiana, los resultados no son determinantes respecto a cuáles de ellos son más sensibles para evaluar el papel de la rizósfera y sus microorganismos asociados en el proceso de descontaminación. No obstante, algunos aspectos deben ser resaltados: a) el tratamiento con pastos mostró cambios en el tiempo para todos los indicadores bioquímicos y microbiológicos evaluados, mientras que el tratamiento sin pastos sólo para la actividad de la deshidrogenasa y el coeficiente metabólico, b) el tratamiento con pastos mostró mayor cantidad de registros en donde la actividad microbiana era mayor o similar al tratamiento sin pastos, pero nunca menor, lo cual probablemente debe estar ligado a la presencia de una rizósfera que favorece dicha actividad y c) por lo general al inicio de los ensayos no se encontraron diferencias entre tratamientos, lo que puede estar ligado 
al proceso de adaptación de la planta recién expuesta a la contaminación y posterior desarrollo del efecto rizosférico. Esto se hace más evidente para respiración basal, carbono microbiano y el coeficiente metabólico.

Por lo antes expuesto, es necesario realizar estudios que incorporen otros indicadores, como por ejemplo, actividades de otras enzimas ligadas a la degradación de hidrocarburos, conteo de microorganismos o biodiversidad de las comunidades microbianas, bioensayos de toxicidad, entre otros, para lograr una mejor interpretación de la información y del papel de las plantas y los microorganismos en la fitorremediación. La meta central de los procesos de remediación en un suelo debe estar orientada no solamente a disminuir la concentración del contaminante y cumplir con un criterio de "limpieza", sino restaurar la salud del mismo, que usualmente se evalúa con variados indicadores físicos, químicos y biológicos (Alkorta et al. 2010, Infante y Morales 2012).

\section{CONCLUSIONES}

Megathyrsus maximus favorece la reducción del contenido de hidrocarburos de suelos contaminados con petróleo extra pesado, en comparación con suelos que no tienen esta planta. No obstante, las diferencias sólo son visibles a partir de los $120 \mathrm{~d}$.

De las fracciones que componen el hidrocarburo extra pesado, disminuyeron las de saturados y aromáticos para el tratamiento con pasto y sólo la de aromáticos para el tratamiento sin pasto, el resto de las fracciones no mostraron cambios significativos, lo cual se asocia a su reconocida baja biodegradabilidad.

El tratamiento con pasto mostró cambios en el tiempo para la respiración basal, carbono microbiano, actividad de la enzima deshidrogenasa y coeficiente metabólico, mientras que el tratamiento sin pasto sólo mostró cambios para la actividad de la enzima deshidrogenasa y el coeficiente metabólico. Por otra parte, el tratamiento con pasto evidenció mayor cantidad de registros en donde la actividad microbiana fue mayor o similar al tratamiento sin plantas, pero nunca menor, lo cual debe estar ligado a la presencia de una rizósfera que favorece dicha actividad.

Las correlaciones lineales entre los diferentes parámetros evaluados sólo fueron significativas y de manera inversa entre el contenido de hidrocarburos y el carbono microbiano y de manera directa entre el contenido de hidrocarburos y el coeficiente metabólico. Ello sugiere que: a) la biomasa microbiana incrementa a expensas de la biodegradación del petróleo como fuente de carbono y energía y b) con la reducción del contenido de los hidrocarburos y probablemente de la toxicidad que estos producen, el ambiente del suelo es menos estresante para los microorganismos y en consecuencia se reduce el costo de mantenimiento de la biomasa microbiana, lo que se traduce en una menor respiración por biomasa de microorganismos.

\section{AGRADECIMIENTOS}

Este proyecto fue financiado por el Fondo Nacional de Ciencias, Tecnología e Investigación de Venezuela. Proyecto Misión Ciencias 2007001401.

\section{REFERENCIAS}

Adams R., Zavala-Cruz J. y Morales F. (2008). Concentración residual de hidrocarburos en suelo del trópico. II. Afectación a la fertilidad y su recuperación. Interciencia 33 (8), 483-489.

Alkorta I., Becerril J. y Garbisu C. (2010). Recovery of soil health: The ultimate goal of soil remediation processes. En: Trends in bioremediation and phytoremediation (G. Plaza, Ed.). Research Signpost, Kerala, India, pp. 1-9.

Alef K. y Nannipieri P. (1995). Methods in applied soil microbiology and biochemestry. Academic Press. Harcourt Brace and Company Publishers, Londres, Inglaterra, $576 \mathrm{pp}$.

Anderson J. e Ingram J. (1992). Tropical soil biology and fertility: A handbook of methods. C.A.B. International, Oxford, Inglaterra, $221 \mathrm{pp}$.

APHA (1998). Standard methods for the examination of water and wastewater. 20a ed. American Public Health Association. Washington, EUA, 1325 pp.

Besalatpour A.A., Hajabbasi M.A. y Khoshgoftarmanesh A.H. (2010). Reclamation of a petroleum-contaminated calcareous soil using phytostimulation. Soil Sediment. Contam. 19 (5), 547-559.

Caravaca F. y Roldán A. (2003). Assessing changes in physical and biological properties in a soil contaminated by oil sludges under semiarid Mediterranean conditions. Geoderma 117 (1-2), 53-61. DOI: 10.1016/S0016-7061(03)00118-6

Casida L. Jr., Klein D. y Santoro T. (1964). Soil dehydrogenase activity. Soil Sci. 98, 371-376.

Chávez-Maldonado E., Rivera-Cruz M.C., IzquierdoReyes F. y Palma-López D.J. (2010). Efectos de rizósfera, microorganismos y fertilización en la biorremediación y fitorremediación en suelos con petróleo crudo nuevo e intemperizado. Universidad y Ciencia $26,121-136$. 
Córdova A., Infante C., López L. y Lugo P. (2013). Efecto de la concentración inicial de crudo en la biorremediación de un suelo contaminado. Revista GEO 44, 115-120.

Ehrmann U., Infante C. y Martínez H. (2009). Correlation between physical chemical properties of different venezuelan crude oils and their biodegradability. Memorias. 24th International Conference on Solid Waste Technology and Management, Philadelphia, EUA. 15 al 18 de marzo de 2009. CD-ROM.

Head I.M., Gray N.D. y Larter S.R. (2014). Life in the slow lane; biogeochemistry of biodegraded petroleum containing reservoirs and implications for energy recovery and carbon management. Front. Microbiol. 5,566 .

DOI: $10.3389 /$ fmicb.2014.00566

Hernández-Valencia I. y Mager D. (2003). Uso de Brachiaria brizantha y Panicum maximum para fitorremediar suelos contaminados con hidrocarburos livianos. Bioagro 15 (3), 149-155.

Hutchinson S.L., Banks M.K. y Schwab A.P. (2001). Phytoremediation of aged petroleum sludge. J. Environ. Qual. 30, 395-403.

DOI: $10.2134 /$ jeq2 $001.302395 \mathrm{x}$

Infante C., Morales F., Ehrmann E.U., Hernández-Valencia I. y León N. (2010a). Hydrocarbons bioremediation and phytoremediation in tropical soils: Venezuelan study case. En: Trends in bioremediation and phytoremediation (G. Plaza, Ed.). Research Signpost, Kerala, India, pp. 429-451.

Infante C., Ortega C., Morales F., Ehrmann U., HernándezValencia I. y Pérez R. (2010b). Efecto del potasio en la biorremediación de un suelo contaminado con un crudo liviano. Bioagro 22 (2),145-152.

Infante C. y Morales F. (2012). Evaluación de la toxicidad en desechos y suelos petrolizados empleando semillas de Lactuca sativa L. Interciencia 37 (1), 1-7.

Infante C., Hernández-Valencia I., López L. y Toro M. (2012). Phytoremediation of petroleum hydrocarbon contaminated soils in Venezuela. En: Phytotechnologies: Remediation of environmental contaminants (N.A. Anjum, M.E. Pereira, I. Ahmad, A.C. Duarte, S. Umar y N.A. Khan, Eds.). CRC Press, Taylor and Francis Group, Boca Raton, EUA, pp. 99-112.

Khan S., Afzal M., Iqbal S. y Khan Q.M. (2013). Plantbacteria partnerships for the remediation of hydrocarbon contaminated soils. Chemosphere 90 (4), 1317-1332.

DOI: 10.1016/j.chemosphere.2012.09.045
Mager D. y Hernández-Valencia I. (2013). Actividad microbiana durante la fitorremediación de un suelo contaminado con un crudo liviano. Rev. Fac. Agron. (LUZ) 30 (1), 52-71.

Marin J.A., Hernandez T. y Garcia C. (2005). Bioremediation of oil refinery sludge by landfarming in semiarid conditions: Influence on soil microbial activity. Environ. Res. 98 (2), 185-195.

DOI: $10.1016 / j$.envres.2004.06.005

Miya R.K. y Firestone M.K. (2001). Enhanced phenanthrene biodegradation in soil by slender oat root exudates and root debris. Environ. Qual. 30 (6), 19111918. DOI: $10.2134 /$ jeq2001.1911

Moubasher H.A., Hegazy A.K., Mohamed N.H., Moustafa Y.M., Kabiel H.F. y Hamad, A.A. (2015). Phytoremediation of soils polluted with crude petroleum oil using Bassia scoparia and its associated rhizosphere microorganisms. Int. Biodeter. Biod. 98, 113-120. DOI: 10.1016/j.ibiod.2014.11.019

Pilon E. (2005). Phytoremediation. Ann Rev. Plant Biol. $56,15-39$.

DOI: 10.1146/annurev.arplant.56.032604.144214

Płaza G., Nałecz-Jawecki G., Ulfig K. y Brigmon R. (2005). The application of bioassays as indicators of petroleum-contaminated soil remediation. Chemosphere 59 (2), 289-296.

DOI: 10.1016/j.chemosphere.2004.11.049

Rengarajan T., Rajendran P., Nandakumar N., Lokeshkumar B., Rajendran P. y Nishigaki I. (2015). Exposure to polycyclic aromatic hydrocarbons with special focus on cancer. Asian Pac. J. Trop. Biomed 5 (3), 182-189. DOI: $10.1016 / \mathrm{S} 2221-1691(15) 30003-4$

Riveroll-Larios J., Escalante-Espinosa E., Focil-Monterrubio R. y Díaz-Ramírez I. (2015). Biological activity assessment in mexican tropical soils with different hydrocarbon contamination histories. Water Air Soil Poll. 226, 353. DOI: 10.1007/s11270-015-2621-1

Statsoft (2007). Statistica. Version 8.0. for Window. Tulsa, OK, EUA.

USEPA (1996). Soxhlet extraction, v. I-B. Method 3540C. United States Environmental Protection Agency, Washington D.C., EUA, 8 pp.

Vance E.D., Brookes P.C. y Jenkinson D.S. (1987). An extraction method of measuring soil microbial biomass C. Soil Biol. Biochem. 19 (6), 703-707. DOI: 10.1016/0038-0717(87)90052-6

Yavari S., Malakahmad A. y Sapari N.B. (2015). A review on phytoremediation of crude oil spills. Water Air Soil Poll. 226, 279. DOI: 10.1007/s11270-015-2550-z 